\title{
EFFICIENT GABOR EXPANSION USING NON MINIMAL DUAL GABOR WINDOWS
}

\author{
Nagesh K. Subbanna and Yonina C. Eldar \\ Department of Electrical Engineering \\ Technion - Israel Institute of Technology \\ Haifa 32000, Israel.
}

\begin{abstract}
In this paper, we illustrate the computational advantages of a class of non minimal dual Gabor frames over the traditional minimal dual Gabor frame. Specifically, we demonstrate that using non minimal dual Gabor frames can lead to Gabor expansions that can be computed far more efficiently than the traditional expansions. Several properties of these non minimal Gabor frames are mentioned and some necessary and sufficient conditions are derived for the existence of this class of non minimal duals.
\end{abstract}

\section{INTRODUCTION}

In many applications, it is important to represent a signal in the time-frequency domain in order to be able to localise the frequencies. Localisation of frequencies is important since many recognition techniques, both biological and computer based, are dependent on the detection of change of frequencies in time. One of the most popular ways to represent a signal in the combined time-frequency domain is the Gabor representation since it has the important features of uniform resloution throughout the combined space and the best possible resolution (with the Gaussian window) [1]. Gabor representation is used in many applications ranging from speech processing and texture segmentation to pattern recognition and object recognition among others.

Given a discrete time signal $f[k]$ of length $L$ the Gabor coefficients are given by:

$$
c_{m, n}=\sum_{k=0}^{L-1} f[k] g^{*}[k-n a] e^{-j 2 \pi m b k / L},
$$

where $a$ is the shift along the time axis, $b$ is the shift along the frequency axis, and $g[k]$ is the window function used. Essentially we project the signal into the combined space using a translated and modulated window function. The reconstruction $f[k]$ from the coefficients $c_{m, n}$ is given by [2]:

$$
f[k]=\sum_{m=0}^{\bar{b}-1} \sum_{n=0}^{\bar{a}-1} c_{m, n} \gamma[k-n a] e^{j 2 \pi m b k / L}
$$

where $\gamma[k]$ is a dual Gabor window, $\bar{a}=L / a \in N$ is the number of shifts along the time axis, $\bar{b}=L / b \in N$ is the number of shifts along the frequency axis and $N$ denotes the set of natural numbers. The task is to efficiently compute a dual Gabor window $\gamma[k]$.

In order for a representation of the form (2) to exist for all sequences $f[k]$ of length $L, a$ and $b$ must satisfy the condition $a b \leq L$ [2], [3], [4]. If $a b=L$, then the representation (2) exists, but it is unstable [4], and the localisation properties are lost [2]. Hence we assume that $a b<L$ in which case the representation is overcomplete, meaning that the set of vectors $\left\{g_{m, n}=g[k-n a] e^{j 2 \pi m b k / L}\right\}$ is linearly dependent. In this case, there are infinite choices for the dual Gabor window $\gamma[k]$. The most popular choice [3] is to find the minimum norm dual of the Gabor window $g[k]$. There are, however, two main drawbacks to this approach. The first problem is that computing the minimal dual can be computationally demanding. The second problem is that for certain choices of the parameters, the minimum norm dual window can become very unstable (this will be shown in Section 5).

In this paper, we show that there are other kinds of duals possible and establish conditions on their existence. We also investigate one particular choice of non minimal Gabor dual that is easy to handle computationally and investigate its properties. We then show that using our technique it is possible to improve upon the stability of the dual gabor window.

The paper is organised as follows: In Section 2, we formulate the problem. In Section 3, we discuss the existence conditions of the non minimal duals, and in Section 4, we present our technique for reducing computational complexity. Finally, in Section 5, we present our simulations and discuss them.

\section{NON MINIMAL DUAL GABOR FUNCTIONS}

We consider the Gabor expansion of discrete-time signals of finite length $L$. Given a set of Gabor vectors $g_{m, n}[k]=$ $g[k-n a] e^{j 2 \pi m b k / L}, k=0,1, \ldots, L-1$ that constitute an overcomplete basis for $C^{L}$, our task is to reconstruct the 
signal $f$ from the coefficients given in (1), which can be written in matrix form as

$$
c=G^{*} f
$$

Here $f=f[k]$ is a vector of length $L$ having elements $f[k]$, $c$ is a vector of length $\bar{a} \bar{b}$ with elements of $c_{m, n}$, and $G$ is an $L \times \bar{a} \bar{b}$ matrix defined by

$$
G=\left[\begin{array}{ccc}
g_{0,0}[0] & \cdots & g_{\bar{a}-1, \bar{b}-1}[0] \\
g_{0,0}[1] & \cdots & g_{\bar{a}-1, \bar{b}-1}[1] \\
\vdots & \vdots & \vdots \\
g_{0,0}[L-1] & \cdots & g_{\bar{a}-1, \bar{b}-1}[L-1]
\end{array}\right] .
$$

We reconstruct $f$ from the coefficients $c$ using (2), where the dual Gabor window satisfies

$$
\Gamma G^{*}=I
$$

Here $\Gamma$ is the matrix of columns $\gamma_{m, n}[k]=\gamma[k-$ $n a] e^{j 2 \pi m b k / L}$. The traditional method is to choose $\Gamma=$ $\left(G^{*}\right)^{\dagger}=\left(G G^{*}\right)^{-1} G$, where $(\cdot)^{\dagger}$ is the pseudo inverse. This corresponds to the minimum norm dual of the Gabor window function used. Since the vectors $g_{m, n}$ span $C^{L}, G G^{*}$ is always invertible. However, the creation of the matrix $G G^{*}$ and computation of its inverse are non trivial tasks and are computationally expensive. In order to overcome this problem, we propose a dual of the form $\left\{d_{m, n}=d[k-n a] e^{j 2 \pi m b k / L}\right\}$ whose vectors span $C^{L}$ and are columns of the matrix

$$
D=\left(H G^{*}\right)^{-1} H,
$$

where $H$ is a matrix that corresponds to an overcomplete basis for $C^{L}$, with vectors $h_{m, n}[k]=h[k-n a] e^{j 2 \pi m b k / L}$ and has the same dimensions as $G$. The advantage here is that since we control $H$ the computational task can be eased out considerably and the properties of the dual window can be tailored to the needs of the user, as we show in Section 4. Further, in Section 5, we show that a correct choice of $H$ can also improve the stability of the expansion.

\section{PROPERTIES OF NON MINIMAL-NORM DUAL}

We first discuss conditions under which a dual of the form (6) exists. A necessary and sufficient condition is given by the following theorem.

Theorem 1 The non minimal-norm dual Gabor frame of the form $D=\left(H G^{*}\right)^{-1} H$ exists iff $R\left(G^{*}\right) \cap N(H)=\{0\}$, where $\mathcal{R}(\cdot)$ is the range and $\mathcal{N}(\cdot)$ is the null space.

Proof : It can be easily seen that if the columns of $H$ span $C^{L}$, then the columns of $D$ also span $C^{L}$ provided the matrix $H G^{*}$ is invertible. So, it is sufficient to show that the matrix $H G^{*}$ is invertible or equivalently, that if $H G^{*} f=0$, then $f=0$. Let us assume that $f \neq 0$. Since the matrix $G$ has a rank of $L$, we know that $c=G^{*} f \neq 0$. Therefore, $H G^{*} f=0$ if and only if $H c=0$ which implies that $c \in N(H)$. In addition, $c \in R\left(G^{*}\right)$, so that if $N(H) \cap R\left(G^{*}\right)=\{0\}$, then $H c \neq 0$, and $H G^{*}$ is invertible. The reverse implication is obvious.

Theorem 1 provides a sufficient condition for the existence of the dual Gabor window of the kind shown in (6). The following theorem gives a necessary condition for the existence of the dual, the proof of which can be found in [5]. Theorem 2 A necessary condition for the matrix $H G^{*}$ to be invertible is given by $\sum_{n=0}^{\bar{a}-1} g^{*}[k-n a+q \bar{b}] h[k-n a] \neq$ $0, q=0, \ldots, b-1, k=0,1, \ldots, L-1$.

Some properties of the matrix $P=\left(H G^{*}\right)$ are mentioned below. All of these properties are also valid for the matrix $S=\left(G G^{*}\right)$. Owing to paucity of space, the proofs have not been given here. Some of the proofs are parallel to the ones found in [3] and others are found in [5].

1. Banded structure of the matrix: The nonzero entries of $P$ are located on the main diagonal and the $k$ th sub-diagonal of $P$ for $k= \pm \bar{b}, \pm 2 \bar{b}, \cdots, \pm(b-1) \bar{b}$. The $P_{k, l}$-th entry is given by $P_{k, l}=\bar{b} \sum_{n=0}^{\bar{a}-1} h[k-$ $n a] g^{*}[l-n a]$ if $|k-l|$ is divided by $\bar{b}$ and 0 otherwise. This shows the extreme sparsity of the matrix.

2. Periodicity of the matrix: Every diagonal and $l \bar{b}, l=1, \ldots, b-1$ sub-diagonal of the matrix $P$ is periodic with period $a$. This property has very important implications in calculating the dual for certain choices of $a$ and $b$ as will be shown later in this section.

3. Block Circulant Structure: If the first $a \times L$ block of the matrix $P$ is $A$, then the $k$-th block is the first block rotated by $(k-1) a$ for $k=1,2, \cdots, \bar{a}-1$. This property shows that the entire matrix can be created by rotating the first $a \times L$ block so that the matrix is block circulant.

Using the properties above, another necessary and sufficient condition can be formulated for the frame of the form $D=\left(H G^{*}\right) H$ to exist. We showed in Theorem 1 that it is enough to prove that $H G^{*}$ is invertible for the frame to exist. Here we give another condition for invertibility of the matrix $H G^{*}$ based on the block circulant property of $H G^{*}$.

The block circulant matrix $P=H G^{*}$ is given by $\mathcal{C}\left(A_{0}, A_{1}, \ldots, A_{\bar{a}-1}\right)$, where $A_{i} \in R^{a \times a}$ and

$P=\mathcal{C}\left(A_{0}, A_{1}, \ldots, A_{\bar{a}-1}\right)=\left[\begin{array}{cccc}A_{0} & A_{1} & \cdots & A_{\bar{a}-1} \\ A_{\bar{a}-1} & A_{0} & \cdots & A_{\bar{a}-2} \\ \vdots & \vdots & \cdots & \vdots \\ A_{1} & A_{2} & \cdots & A_{0}\end{array}\right]$ 
The discrete Fourier transform of $P$ is given by $\mathcal{F}(P)=$ $\mathcal{C}\left(\tilde{A_{0}}, \tilde{A_{1}}, \ldots, \tilde{A_{\bar{a}}-1}\right)$ where

$$
\tilde{A}_{s}=\sum_{r=0}^{\bar{a}-1} e^{-j 2 \pi r s / \bar{a}} A_{r} .
$$

Lemma 1: [6] The matrix $P$ is invertible if and only if $\tilde{A}_{s}, s \in 0,1, \ldots, \bar{a}-1$ is invertible for every $s$, where $\tilde{A}_{s}$ is as defined in (8).

\section{COMPUTATIONAL ISSUES}

The minimum dual requires computing the matrix $S=$ $G G^{*}$ whose $k j$-th element is given by [3] $S_{k, j}=$ $\bar{b} \sum_{n=0}^{\bar{a}-1} g[k-n a] g^{*}[j-n a]$ if $|k-j|=q \bar{b}, q=0, \ldots, b-$ 1 and 0 otherwise. The number of operations necessary to carry out the entire generation of the matrix is $(\bar{a}+1) a b$ multiplications and $b a \bar{a}$ additions. In many cases, $\bar{a}$ is very large since we do not shift the window too far. The shift needs to be small enough to be able to capture all the details of the signal accurately. Therefore, anything that removes this component from the multiplications is going to simplify the procedure greatly. As we will show, this can be achieved by a proper choice of $H$. The function $g[k]$ is usually chosen to be the Gaussian function $g[k]=e^{-k^{2} / \sigma_{1}^{2}}, \sigma_{1}>0$.

The elements of $H G^{*}$ are theoretically generated as shown in property 1 , but because we have the freedom to choose $h[k]$, we control $H$ and have a far faster technique to generate $H G^{*}$. In this case, $h[k]=e^{-k^{2} / \sigma_{2}^{2}}$ can also be chosen as a Gaussian function. We select $\sigma_{2}$ such that the effective spread of the $h[k]$ is $a$. In this it is to be understood that outside this effective spread, the value of the function is not equal to zero. Rather it is made negligibly small (less than a thousandth or even less of the value of the function at the peak).

Theorem 4: If $g[k]=e^{-k^{2} / \sigma_{1}^{2}}$ and $h[k]=e^{-k^{2} / \sigma_{2}^{2}}$ and $\bar{b}$ is divisible by $a$, then the matrix $P=H G^{*}$ is invertible.

Proof: From Lemma 1, we know that $P$ is invertible iff all the discrete Fourier blocks $\tilde{A}_{s}$ are invertible. From property 1 , we know that the non-zero elements of $P$ are only on the principal diagonal and the sub-diagonal $k \bar{b}, k=$ $0,1, \ldots, b-1$ and the discrete Fourier transform of $P$ is given by $\mathcal{C}\left(\tilde{A}_{0}, \tilde{A}_{1}, \ldots, A_{\tilde{a}-1}\right)$ where $\tilde{A}_{s}$ is defined as in (8). The blocks $A_{r}$ are of size $a \times a$ and since $\bar{b}$ is divisible by $a$, we can see from this that every $A_{r}$ is either a diagonal matrix or a Zero matrix and the discrete Fourier transform blocks $\tilde{A}_{s}$ is a diagonal matrix and is given by

$\tilde{A}_{s}=1 . A_{0}+e^{-j 2 \pi \bar{b} s / a \bar{a}} A_{\bar{b} / a}+\cdots+e^{-j 2 \pi(b-1) \bar{b} s / a \bar{a}} A_{(b-1) \bar{b} / a}$

Now if we can show that the diagonal elements of the Fourier block matrix is not zero out proof is complete. The diagonal elements of $\tilde{A}_{s}$ are given by

$$
\begin{aligned}
A_{s}(k, k)= & \bar{b} \sum_{q=0}^{b-1} \sum_{n=0}^{\bar{a}-1} e^{-(k-n a)^{2} / \sigma_{2}^{2}} e^{-(k-n a+q \bar{b})^{2} / \sigma_{1}^{2}} e^{-j 2 \pi q \bar{b} s / a \bar{a}} \\
& =\bar{b} \sum_{q=0}^{b-1} \sum_{n=0}^{\bar{a}-1} e^{-(k-n a)^{2} / \sigma_{2}^{2}} e^{-(k-n a+q \bar{b})^{2} / \sigma_{1}^{2}} e^{-j 2 \pi q s / b} \\
& =\bar{b} \sum_{q=0}^{b-1} e^{-j 2 \pi q s / b} \sum_{n=0}^{\bar{a}-1} e^{-(k-n a)^{2} / \sigma_{2}^{2}} e^{-(k-n a+q \bar{b})^{2} / \sigma_{1}^{2}}
\end{aligned}
$$

It can easily be seen that (10) cannot be zero. Therefore, the statement is proved.

The effective spread of the function $h[k]$ is from $-a / 2$ to $a / 2$. Therefore, the matrix $H$ is made block diagonal for all practical purposes and looks as shown below.

$$
H=\left[\begin{array}{cccc}
B & \varepsilon & \cdots & \varepsilon \\
\varepsilon & B & \cdots & \varepsilon \\
\cdots & \ldots & \cdots & \\
\varepsilon & \varepsilon & \cdots & B
\end{array}\right]
$$

The $\varepsilon$ is used to denote that the value of the elements in these blocks is extremely small. Each block $B$ is of size $a \times a$ and this is true for the $\varepsilon$ blocks as well.

In the creation of the matrix $P=H G^{*}$ it can be seen that, because we limited the spread of $h[k]$ the $k, k+q \bar{b}$-th elements of $P$ are generated by $P_{k, k+q \bar{b}}=$ $\bar{b} e^{-(k-n a)^{2} / \sigma_{2}^{2}} e^{-(k+q \bar{b}-n a) / \sigma_{1}^{2}}, q \in 0, \ldots, b-1$ and $k-$ $n a \in 0,1, \ldots, a$ and 0 otherwise.

The number of operations to generate this matrix is $2 a b$ and the number of additions necessary is 0 . This is the greatest advantage in this method.

The algorithm for calculating the inverse of the matrix is usually the Conjugate Gradients algorithm. Utilising the sparse structure of the matrix, the conjugate algorithm can do a very good job in calculating the inverse of the matrix $H G^{*}$ (it has a complexity of $O(n \log (n))$ ).

\section{SIMULATIONS AND DISCUSSION}

The simulations show a Gaussian window and two of its possible dual functions. Figure 1 shows a typical Gaussian window that has $\sigma_{1}=4$. The lattice constants $(a, b)$ were both set to 4 and the length of the signal was 64 . Figure 2 shows the minimum norm dual and Figure 3 shows the non minimum dual with $\sigma_{2}=2$. As can be seen, even the non minimal dual function gives a good localisation. The number of operations necessary to compute the two duals can be computed as given in Section 4. Due to our choice of $H$ in place of the traditional $G$, the number of flops (multiplication operations only) necessary to compute the dual is about $1 / 3$ of what would be necessary to compute the minimum 


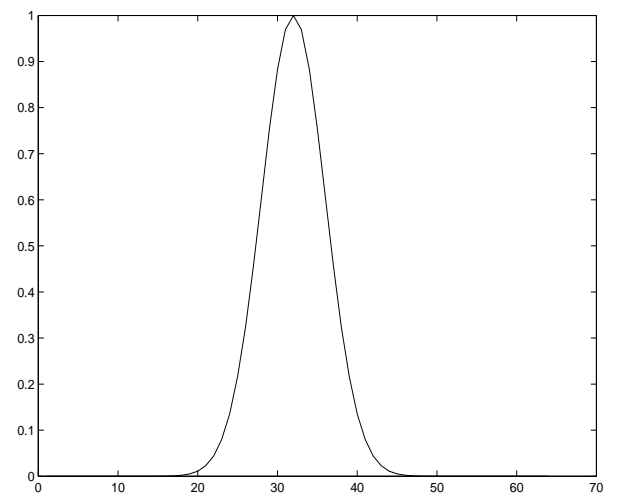

Fig. 1. The Gaussian window with $\sigma_{1}=4$

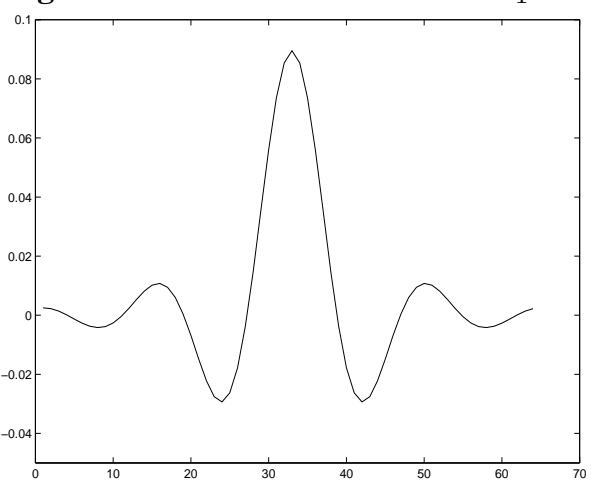

Fig. 2. The minimum dual window for lattice constants $\left(a=4, b=8, \sigma_{1}=\sigma_{2}=4\right)$

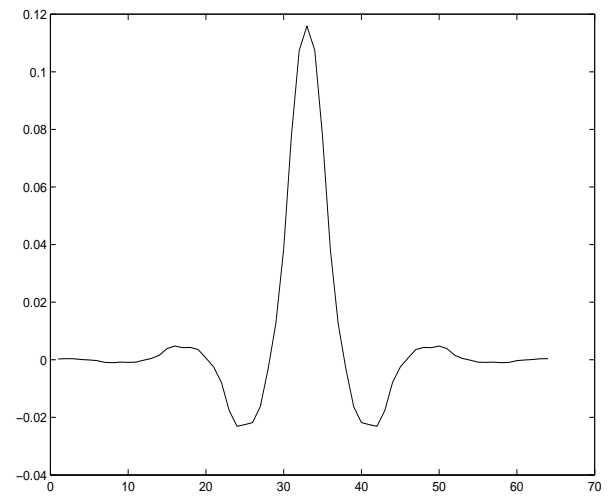

Fig. 3. The non minimum dual window for lattice constants $\left(\mathrm{a}=4, \mathrm{~b}=8, \sigma_{1}=4, \sigma_{2}=2\right)$ dual. As the length of the sequence and the number of shifts goes up, the number of operations saved (multiplications and additions) will increase greatly.

\subsection{Value of the Determinant}

There are many cases where the choice of parameters is such that computing the inverse of $G G^{*}$ will be impossible. In many pattern recognition applications when it is necessary to recognise low frequencies, the shift $(a)$ has to be small and the spread of the function $\left(\sigma_{1}\right)$ has to be large. It has been observed that when the shift and the spread are too different, the value of the determinant of $G G^{*}$ will be very close to 0 (although it is never technically equal to 0 ). For instance, if $a=16$ and $\sigma_{1}=80$, the determinant would be very small (of the order of $10^{-59}$ ). In such a case, the matrix $G G^{*}$ will be nearly singular and it is impossible to compute the inverse of such a matrix. Our method provides a means to overcome this trap. By selecting $\sigma_{2}$ (the spread of $h[\cdot]$ ) different from $\sigma_{1}$ (the spread of $g[\cdot]$ ), it is possible to obtain a larger determinant and consequently have a dual function for the window function with the set of parameters given. So, it is of interest to see how the value of the determinant changes when the values of the spread of the window functions $(g[\cdot]$ and $h[\cdot])$ change. The full graphs are shown in [5].

\section{REFERENCES}

[1] D. Gabor, "Theory of Communications", Journal IEE (London), 93(III): 429-457, November 1946.

[2] J. Wexler and S. Raz "Discrete Gabor Expansions", Signal Processing, 21(3):pp 207-221, November 1990.

[3] S. Qiu and H. Feichtinger, "Discrete Gabor Structures and Optimal Representation", IEEE Transactions on Signal Processing, 43(10):pp 2258-2268, October 1995.

[4] M. Zibulski and Y. Y. Zeevi, "Oversampling in the Gabor scheme", IEEE Transactions on Signal Processing, 41(8): pp 2679-2687, August 1993.

[5] N. K. Subbanna and Y. C. Eldar, “Efficient algorithm to compute dual Gabor windows", in preparation.

[6] A. Beck, Y. C. Eldar, and A. Ben Tal, "Minmax Mean Squared Error Estimation of multichannel signals", preprint 